\title{
Patients with Multifocal Macroscopic Papillary Thyroid Carcinoma Have a Low Risk of Recurrence at Early Follow-Up after Total Thyroidectomy and Radioactive lodine Treatment
}

\author{
Amanda La Greca ${ }^{a}$ Bin Xu ${ }^{b}$ Ronald Ghossein ${ }^{b}$ R. Michael Tuttle ${ }^{a}$ \\ Mona M. Sabra ${ }^{a}$ \\ ${ }^{a}$ Endocrinology Service, Department of Medicine, and ${ }^{b}$ Department of Pathology, Memorial Sloan-Kettering \\ Cancer Center and Weill-Cornell College of Medicine, New York, N.Y., USA
}

\section{Key Words}

Thyroid cancer recurrence - Multifocal macroscopic

papillary thyroid cancer $\cdot$ Clinical outcomes

\begin{abstract}
Background: Multifocal thyroid cancer involvement is a common presentation in papillary thyroid cancer. The risk of recurrence of intrathyroidal multifocal papillary microcarcinoma $(<1 \mathrm{~cm})$ is documented to be low. However, the risk of recurrence of multifocal macroscopic thyroid cancer is not known. Prior studies have suggested that both the number of foci and the presence of nodal involvement at diagnosis are important predictors of recurrence in multifocal papillary thyroid carcinoma (PTC). Objectives: In this retrospective review of 99 patients presenting with multifocal macroscopic PTC (with 2 tumor foci $>1 \mathrm{~cm}$ ) without gross extrathyroidal extension, we examined the clinical outcomes of patients in the first 2 years after the initial therapy and at the end of the follow-up period (median: 5 years). Results: Half of the patients presenting with multifocal macroscopic PTC had nodal involvement at diagnosis. Only 4 patients had a recurrence
\end{abstract}

\section{KARGER}

(C) 2016 European Thyroid Association

Published by S. Karger AG, Basel

E-Mail karger@karger.com

www.karger.com/etj on long-term follow-up, all with classic or tall-cell variant PTC with bulky nodal involvement at diagnosis. The number of tumor foci did not influence the risk of recurrence in this cohort. The median time to recurrence in these 4 patients was 11 years, with all patients having a recurrence after 9 years of follow-up. None of patients developed distant metastasis or died from thyroid cancer. Conclusions: Patients presenting with multifocal macroscopic papillary thyroid cancer without bulky nodal involvement or gross extrathyroidal extension have a low risk of thyroid cancer recurrence.

(c) 2016 European Thyroid Association Published by S. Karger AG, Basel

\section{Introduction}

Multifocal involvement of the thyroid gland is a wellrecognized feature of papillary carcinoma. The reported frequency is about $20 \%$, with wide variations depending on the extent of the sampling and reporting by the pa-

Amanda La Greca and Bin Xu share first authorship of this paper.
Mona M. Sabra, MD

Memorial Sloan Kettering Cancer Center 1275 York Avenue

New York, NY 10065 (USA)

E-Mail sabram@mskcc.org 
thologist [1-3]. Multifocal papillary thyroid carcinomas (PTC) can be divided into 2 categories depending on the tumor size: multifocal papillary microcarcinoma (all of the lesions sized $<1 \mathrm{~cm}$ ) and multifocal macroscopic papillary thyroid cancer (includes more than 1 tumor focus sized $\geq 1 \mathrm{~cm}$ ).

Several studies have confirmed the risk of structural disease recurrence in multifocal papillary microcarcinoma treated with total thyroidectomy to be low, varying from 4 to 6\% [4-6]. In similar patients, Baudin et al. [7] demonstrated that the number of histologic foci and the extent of surgery significantly influenced the risk of recurrence in multivariate analysis. As such, the recurrence rate for patients with unifocal thyroid microcarcinoma was $1.2 \%$ compared to $8.6 \%$ for patients with multifocal thyroid microcarcinoma $(\mathrm{p}=0.002)$ [7]. Other large retrospective studies have failed to demonstrate that multifocality is a risk factor for thyroid cancer recurrence $[8,9]$.

Given the calculated low risk of recurrence of multifocal papillary thyroid microcarcinoma after total thyroidectomy, routine radioactive iodine (RAI) ablation is not recommended and close surveillance is advocated [10]. In the updated 2015 American Thyroid Association guidelines, the 3-tiered categorical system (i.e. low, intermediate, and high risk of recurrence) did not specifically address the outcomes of and management approach for patients presenting with multifocal macroscopic papillary thyroid cancer [10]. In the absence of studies estimating the risk of structural recurrence in these patients, the treating physician often errs on the side of caution, electing to administer RAI ablation to patients presenting with multifocal macroscopic papillary thyroid cancer and thus potentially providing unnecessary treatment and potentially increasing the risk of treatment-related morbidity.

In this study, we investigated the risk of structural disease recurrence in patients with multifocal macroscopic papillary carcinoma as an independent (prognostic) factor. We hypothesized that the risk of recurrence of multifocal macroscopic papillary thyroid cancer in the absence of other aggressive features, such as gross extrathyroidal extension and bulky nodal involvement, is low and that routine RAI therapy is not required when treating these patients. Our primary end point was the presence of persistent/recurrent structural disease after the initial therapy and at the final follow-up in patients with multifocal PTC with at least 2 tumors, all with a longest diameter of at least $1 \mathrm{~cm}$.

\section{Materials and Methods}

\section{Patient Selection}

After obtaining institutional review board approval, we reviewed the electronic medical records of 124 subjects with multicentric thyroid carcinoma treated at the Memorial Sloan-Kettering Cancer Center between 1985 and 2002 and 2008 and 2015. Accurate determination of multifocality on pathologic examination was not systematically collected at our center for patients diagnosed between 2002 and 2008. These patients were not included in the analysis. Patients were included if they met all of the following conditions: (1) was treated with at least a total thyroidectomy with or without lymph node dissection, (2) had a diagnosis of PTC on surgical pathology, (3) had 2 or more thyroid cancer foci greater than $1 \mathrm{~cm}$ in longest diameter; (4) had at least a 6-month follow-up after surgery, (5) had appropriate follow-up with unstimulated thyroglobulin $(\mathrm{Tg})$ and $\mathrm{Tg}$ antibodies (TgAb) and imaging (neck ultrasounds, CT/MRI, and RAI scanning).

The exclusion criteria were: (1) patients with follicular, Hürthle cell, poorly differentiated, medullary or anaplastic thyroid cancer; (2) patients undergoing lobectomy alone; (3) patients with gross extrathyroidal extension on the surgical specimen; (4) patients without appropriate follow-up, and (5) patients with chronic TSH elevation. From 124 potential patients, 99 patients were selected for this study.

\section{Histopathologic Analysis}

Tumors were classified according to the latest World Health Organization criteria, with the exception of tall-cell variant (TCV) PTC [11]. Tumors were classified as TCV PTC if they contained $>50 \%$ tall cells. The latter cell type was defined as having a height at least twice its width, with oncocytic cytoplasm [12]. All cases were diagnosed by subspecialized head and neck pathologists with expertise in thyroid neoplasia.

\section{Patient Follow-Up}

Patients were followed every 6 months during the first year and then at 6-to 12-month intervals thereafter at the discretion of the treating physician. The frequency of the follow-up visits was based on the estimated risk of recurrence for each individual patient and the clinical course of the disease.

\section{Clinical End Points}

Each patient was risk stratified according to the seventh edition of the American Joint Committee on Cancer TNM (AJCC/TNM) staging system (stage I, II, III, or IV) and the American Thyroid Association (ATA) risk of recurrence stratification system (low, intermediate, or high) $[10,13]$. Clinical outcomes were then assessed at 2 time intervals: best response to the initial therapy and status at the final follow-up. The best clinical response to the initial therapy was assessed based on clinical data obtained in the first 2 years of follow-up using a restratification model proposed and validated by our group $[14,15]$. The definitions of clinical response varied depending on whether the patient was treated with RAI ablation or not. Specifically, patients who received a total thyroidectomy and RAI ablation were classified as having: (1) an excellent response if they achieved a nonstimulated Tg level $<0.2 \mathrm{ng} / \mathrm{ml}$ or undetectable $\mathrm{TgAb}$ and negative imaging (normal neck ultrasound and/or cross-sectional imaging such as CT or MRI and RAI scanning), (2) an indeterminate response if they achieved an unstimulated $\mathrm{Tg}$ level between 0.2 and $1 \mathrm{ng} / \mathrm{ml}$ or TgAb levels were stable 
or declining (in the absence of structural or functional disease) or had nonspecific findings on imaging studies or a faint uptake in the thyroid bed on RAI scanning, (3) a biochemical incomplete response if they achieved a nonstimulated Tg level $>1 \mathrm{ng} / \mathrm{ml}$ or increasing TgAb levels and nonsuspicious imaging findings, or (4) a structural incomplete response if they had structural evidence of disease regardless of $\mathrm{Tg}$ or $\mathrm{TgAb}$ levels. Patients who received a total thyroidectomy without RAI ablation were classified as having: (1) an excellent response if they achieved a nonstimulated $\mathrm{Tg}$ level $<0.2 \mathrm{ng} / \mathrm{ml}$ or undetectable TgAb and negative imaging (normal neck ultrasound and/or cross-sectional imaging such as CT or MRI and RAI scanning), (2) an indeterminate response if they achieved a nonstimulated Tg level between 0.2 and $5 \mathrm{ng} / \mathrm{ml}$ or $\mathrm{TgAb}$ levels were stable or declining (in the absence of structural or functional disease) or they had nonspecific findings on imaging studies or a faint uptake in the thyroid bed on RAI scanning, (3) a biochemical incomplete response if they achieved a nonstimulated Tg level $>5 \mathrm{ng} / \mathrm{ml}$ or increasing Tg values over time with similar TSH levels or increasing TgAb levels and negative imaging, or (4) a structural incomplete response if there was structural or functional evidence of disease regardless of the Tg or TgAb levels [15].

The clinical status at the time of the last follow-up evaluation was recorded for each patient [no clinical evidence of disease, indeterminate response (using the same criteria as above), biochemical persistent disease, structural persistent disease, recurrent disease, or thyroid-related death]. 'No evidence of disease' referred to patients without biochemical or structural evidence of disease. None of the patients had persistent elevated Tg or rising antithyroglobulin antibodies without a structural correlate (biochemical persistent disease). In all of the patients who had a recurrence, the site of recurrence was the neck, and none of the patients developed distant metastasis. The Tg cutoffs used to assess the final clinical status were similar to those used for the initial response to therapy.

\section{Laboratory Studies}

Between 1990 and 1997, various Tg assays with functional sensitivities of approximately $1 \mathrm{mg} / \mathrm{ml}$ were used. Starting in 1998, all Tg values were measured using the DYNOtest-TgS immunoradiometric assay (Brahms Inc., Berlin, Germany; functional sensitivity: $0.6 \mathrm{ng} / \mathrm{ml}$ normalized to Certified Reference Material 457) [16]. $\mathrm{TgAb}$ levels were measured using a Siemens Immulite $2500 \mathrm{Chem}$ istry Analyzer (Siemens Healthcare Diagnostics Inc., Deerfield, Ill., USA). Given the variability of Tg assay detection levels over time, we generally assumed that an excellent response was achieved if the stimulated Tg level was $<1 \mathrm{ng} / \mathrm{ml}$ with undetectable anti-Tg antibodies. If this was not available, we considered a nonstimulated Tg level below the detection level of that specific assay as enough indication of an excellent response. Using this definition, we recognize that a few patients diagnosed before 1998 may have been misclassified as having an excellent response to the initial therapy rather than an indeterminate response.

\section{Statistical Methods}

Continuous data are presented as means \pm SD or medians (range), as appropriate for each variable. Analyses were performed using SPSS software (version 24; SPSS, Inc., Chicago, Ill., USA).

The primary end point was the presence of persistent/recurrent structural disease after the initial therapy and at the final follow-up in patients with multicentric PTC when at least 2 foci measured $1 \mathrm{~cm}$ or more in longest diameter.

Recurrence in Multifocal Papillary

Thyroid Cancer

\section{Results}

\section{Clinicopathologic Characteristics of the Cohort}

The clinicopathologic characteristics of the 99 patients included in this analysis are summarized in table 1 . The patients were a median of 47 years old at the time of the cancer diagnosis, and they had a median of 2 additional foci of thyroid cancer. The median size was $2 \mathrm{~cm}$ for the largest tumor and $1.3 \mathrm{~cm}$ for the second biggest tumor. Classic PTC was the histology of the largest tumor in more than half of the sample (55\%), followed by tall cell (31\%) and follicular variant (13\%). The histology of the second biggest focus was more likely to be similar to that of the primary tumor in classic and follicular variant PTC but not in TCV PTC. Thus, the concordance in histology between the largest and second biggest tumors was 63\% in classic PTC, $50 \%$ in follicular variant PTC, and $19 \%$ in TCV PTC. Twenty-eight percent of the largest tumors were totally encapsulated and $17 \%$ displayed vascular invasion.

All of the patients were treated with a total thyroidectomy. Neck dissection, either central or lateral, was performed only if suspicious nodes were detected by imaging ahead of the surgery and intraoperatively. Thus, none of the studied patients had a prophylactic neck dissection. Half of the patients with multifocal macroscopic PTC had nodal involvement (55\%), with 45 patients with N1a disease (median: 3 affected nodes) and 38 patients with $\mathrm{N} 1 \mathrm{~b}$ disease (median: 4 affected nodes). The median size of the largest metastatic node was $1.4 \mathrm{~cm}$, and extranodal extension was observed in $20 \%$ of the affected nodes.

Five patients were found to have metastasis to the lungs at the time of the cancer diagnosis. Those patients were more likely to be women (80\%) with a median age of 38 years, with larger primary tumors (median size: $3.5 \mathrm{~cm}$ ) and significant nodal disease in the central ( 5 patients, median: 3 affected nodes) and/or lateral compartments (4 patients, median: 9 affected nodes) with extranodal extension (seen in 4 patients). All of these patients had lung micrometastasis (lung nodules $<1 \mathrm{~cm}$ ). Two were detected on diagnostic radioiodine scan at the initial therapy, and 3 were detected by a posttherapy scan and confirmed with a CT scan. At the final follow-up, 4 patients had persistent stable structural disease and 1 patient had no evidence of disease. None of the patients developed distant metastasis during the course of the follow-up.

The majority of the patients received RAI ablation ( $\mathrm{n}=74 ; 75 \%$ ), with a median activity of $145 \mathrm{mCi}$. The administered activity was not known in 5 of these patients. Nine patients received more than one RAI activity during 
Table 1. Clinical characteristics of the cohort $(n=103)$

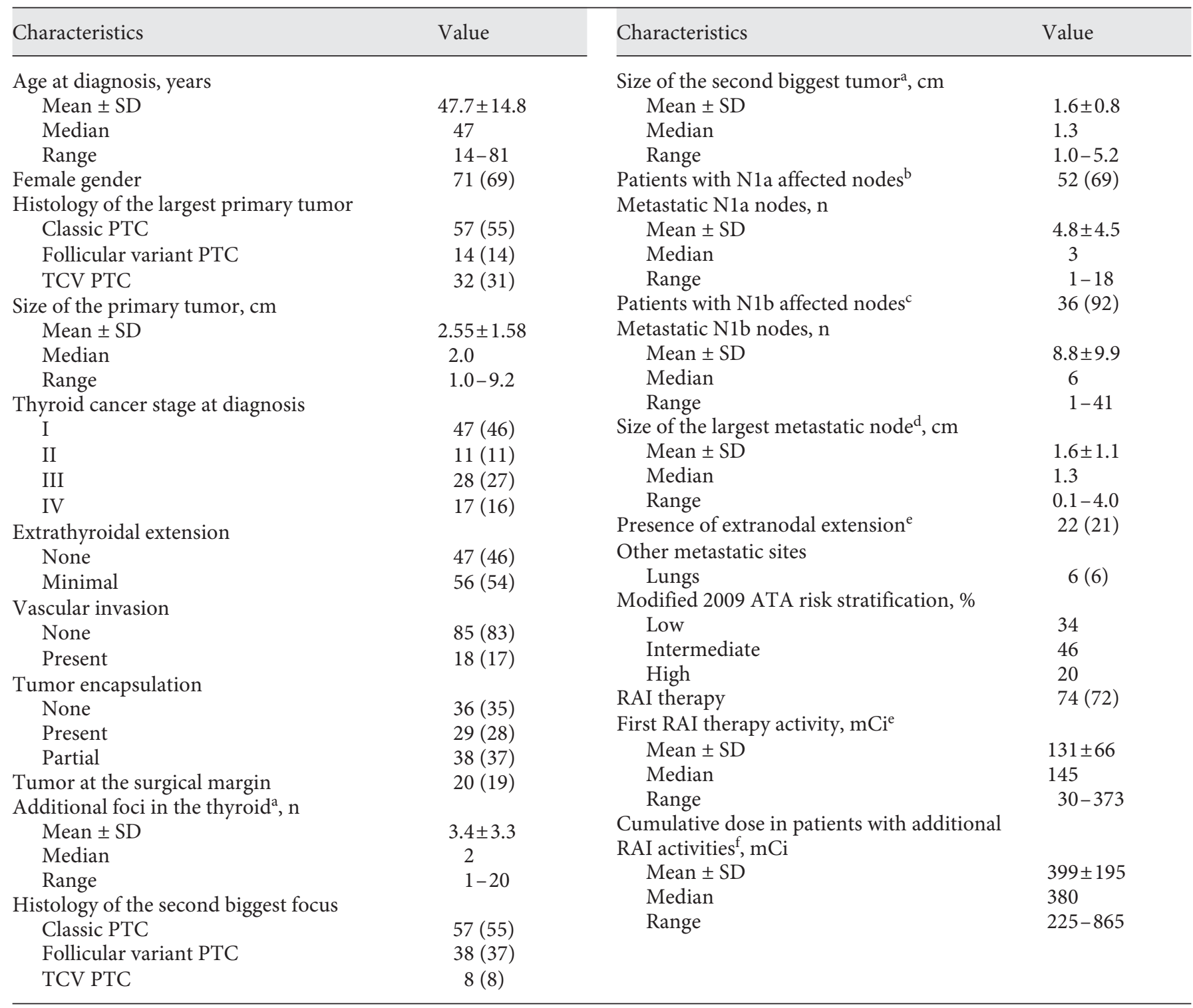

Values are presented as numbers (\%) unless otherwise stated. ${ }^{a} \mathrm{n}=92 .{ }^{\mathrm{b}} \mathrm{n}=75 .{ }^{\mathrm{c}} \mathrm{n}=39 .{ }^{\mathrm{d}} \mathrm{n}=57 ; 5$ patients had an unknown initial dose. ${ }^{\mathrm{e}} \mathrm{n}=43 .{ }^{\mathrm{f}} \mathrm{n}=69$.

the course of their follow-up (7\%), and the median cumulative activity for these 9 patients was $380 \mathrm{mCi}$ (mean: 399 \pm 195 , range $225-865$ ).

The patients were followed for a median of 3 years (range 0.12-29.2). After excluding for patients with gross extrathyroidal extension at the time of thyroid cancer diagnosis, none of the patients with multifocal macroscopic thyroid cancer developed distant metastasis on followup or succumbed to their disease.

\section{Clinical Outcomes}

The clinical outcomes of the patient cohort are summarized in figure 1 . Of the 99 patients included in the final analysis, 5 had recurrences of thyroid cancer. All 5 patients had nodal involvement at diagnosis and none had distant metastasis at diagnosis or during the course of their follow-up. Thus, all recurrences were in the neck. The clinical characteristics and outcomes of these patients are summarized below. 


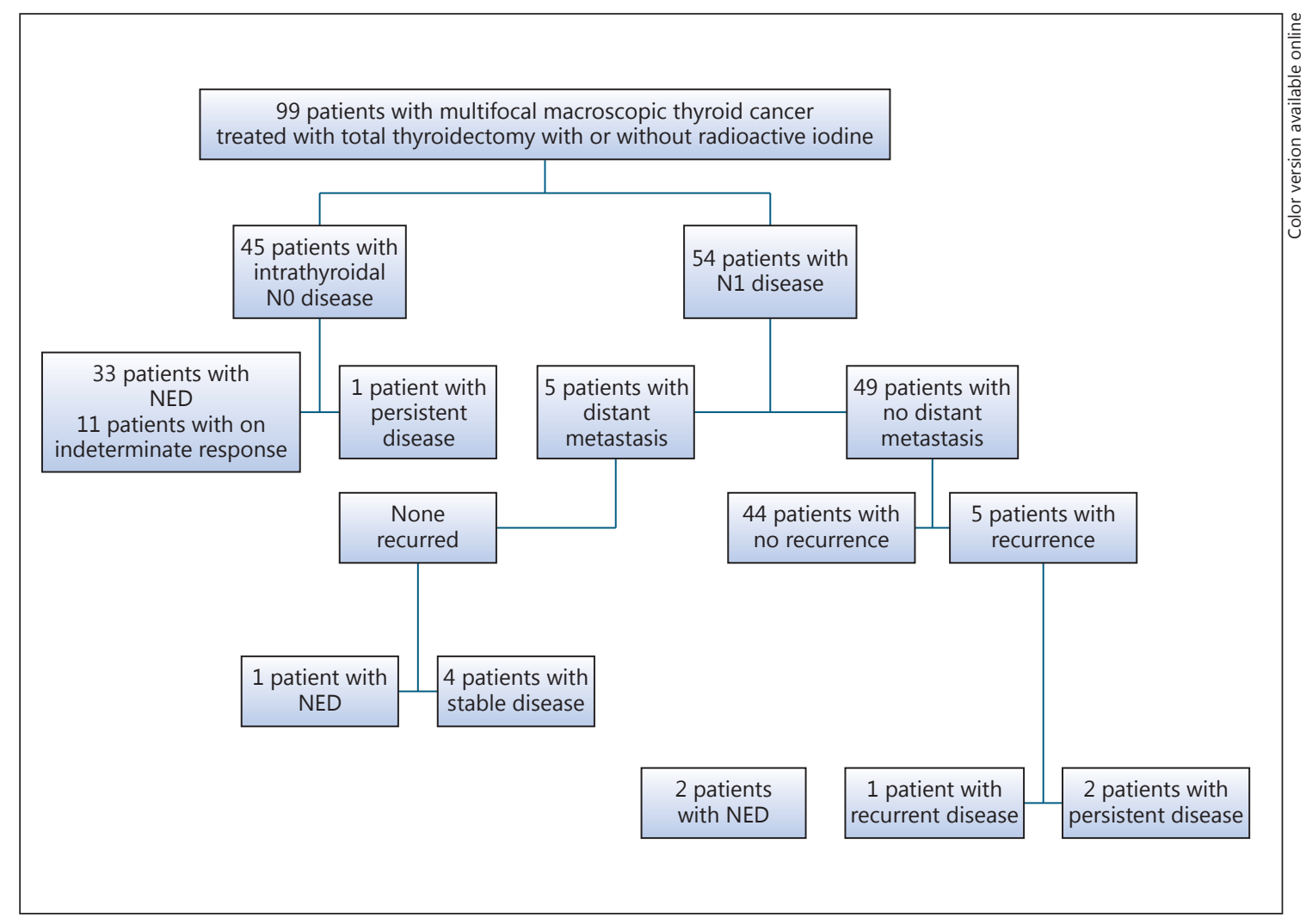

Fig. 1. Clinical outcomes of treated patients with multifocal macroscopic thyroid cancer. NED = No evidence of disease.

Clinical Response Based on the Initial ATA Risk

Stratification

Using the 2009 Modified ATA risk classifier, 34\% of the patients were classified as low risk, $46 \%$ as intermediate risk, and $19 \%$ as high risk. In the first 2 years after the initial therapy, $37 \%$ achieved an excellent response, $45 \%$ achieved an indeterminate response, $7 \%$ achieved a biochemical incomplete response, and $8 \%$ had a structural incomplete response. At the end of the follow-up period (median: 3 years), 60\% of the patients had no evidence of disease, $7 \%$ of the patients had persistent disease, and only 1 patient (1\%) had recurrent disease (table 2 ). A third of the patients had an indeterminate response with undetectable Tg levels and/or detectable but decreasing antithyroglobulin antibodies and/or stable nonspecific thyroid bed or lung nodules.

Interestingly, none of the 34 patients with ATA lowrisk thyroid cancer had long-term recurrences of thyroid cancer. Two of the 19 patients with ATA high-risk thyroid cancer at diagnosis had a recurrence, and 2 of the 46 patients with an ATA intermediate risk at diagnosis had a long-term recurrence of thyroid cancer. All of the patients who had a recurrence had nodal involvement at diagnosis.

A total of 74 patients had follow-up over 1 year. Their risk stratification and outcomes are summarized in table 3. Five patients $(6 \%)$ had a recurrence during disease follow-up, with almost all recurrences occurring 5 years or later after the thyroid cancer diagnosis. At the final follow-up (median: 5 years), similarly to the overall cohort, more than half of the patients had no evidence of disease (55\%), 10\% had persistent stable disease, 34\% had achieved an indeterminate response, and 1 patient had recurrent disease.

\section{Outcomes of Patients Who Received RAI Ablation}

Compared to Those Who Did Not

Seventy-four patients received RAI ablation. Five of these patients had a recurrence on follow-up. The clinical characteristics of these 5 patients are summarized below. Seven of the 74 patients who received RAI ablations were treated with additional RAI therapies; in the 
Table 2. Clinical outcomes of all of the patients $(n=103)$

\begin{tabular}{lc}
\hline Time from diagnosis to the last follow-up, years & \\
Mean \pm SD & $5.7 \pm 6.0$ \\
Median & 3.2 \\
Range & $0.3-29.1$ \\
Best response to the initial therapy & \\
Excellent & $44(38)$ \\
Biochemical incomplete & $7(7)$ \\
Structural incomplete & $8(8)$ \\
Indeterminate & $45(47)$ \\
Recurrence during the course of follow-up & $5(5)$ \\
Clinical outcomes at the end of follow-up & \\
No evidence of disease & $61(61)$ \\
Structurally persistent disease & $6(6)$ \\
Recurrent disease (neck) & $1(1)$ \\
Indeterminate & $31(31)$ \\
No thyroid cancer-related death &
\end{tabular}

Values are presented as numbers (\%) unless otherwise stated. ${ }^{\mathrm{a}} \mathrm{n}=97 .{ }^{\mathrm{b}} \mathrm{n}=99$.

first 2 years after the initial therapy, 1 had an excellent response, 1 achieved an indeterminate response, 1 achieved a biochemical incomplete response, and 4 had a structural incomplete response. At the end of the follow-up and with interim repeat RAI therapy, 2 had no evidence of disease, 2 achieved an indeterminate response, and 3 had stable persistent thyroid cancer. None of these 7 patients had neck recurrences or developed metastasis on follow-up.

At the discretion of the treating physician, 25 patients were initially treated with active surveillance without RAI ablation after the initial surgery. Only 3 of these patients had N1 disease, with up to 3 affected nodes measuring up to $6 \mathrm{~mm}$. Four of these 25 patients were subsequently treated with RAI with a median activity of $92 \mathrm{mCi}$ (mean: $102 \pm 34 \mathrm{mCi}$, range 75-150). None of these patients had a recurrence during the course of their follow-up. Eighteen of these 25 patients had no evidence of disease, while 7 achieved an indeterminate response.

\section{Clinical Characteristics of the Patients with \\ Recurrences}

Tables 4 and 5 describe the clinical characteristics of the 5 patients who had a recurrence during the course of follow-up. All of the patients received an initial RAI ablation. The median size of the primary tumor was $2.7 \mathrm{~cm}$; focal vascular invasion ( $<4$ invasive foci) was only noted
Table 3. Clinical outcomes including patients with follow-up of more than 1 year $(n=79)$

$\begin{array}{lc}\text { Time from diagnosis to the last follow-up, years } & \\ \text { Mean } \pm \text { SD } & 7.3 \pm 6.4 \\ \text { Median } & 5.0 \\ \text { Range } & 1.0-29.2 \\ \text { Modified 2009 ATA risk stratification } & 22(28) \\ \text { Low } & 36(46) \\ \text { Intermediate } & 21(26) \\ \text { High } & \\ \text { Best response to the initial therapy } & 33(42) \\ \text { Excellent } & 8(10) \\ \text { Biochemical incomplete } & 10(13) \\ \text { Structural incomplete } & 28(35) \\ \text { Indeterminate } & 5(6) \\ \text { Recurrence during the course of follow-up } & \\ \text { Clinical outcomes at end of follow-up } & 68(86) \\ \text { No evidence of disease } & 10(13) \\ \text { Persistent disease } & 1(1) \\ \text { Recurrent disease (neck) } & 3(4) \\ \text { Thyroid cancer-related death } & \end{array}$

Values are presented as numbers (\%) unless otherwise stated.

in 1 case, and 3 cases had minor extrathyroidal extension and only 1 case had positive margins on surgical resection. The median size of the separate foci was $1.3 \mathrm{~cm}$. Four out of 5 patients had bulky nodal involvement in the lateral neck with extranodal extension at diagnosis.

The best response to the initial therapy in those $5 \mathrm{pa}$ tients was excellent $(\mathrm{n}=1)$, biochemical incomplete $(\mathrm{n}=$ $3)$, and indeterminate $(\mathrm{n}=1)$. One patient with an initial biochemical incomplete response presented with structural recurrence 2 years after the initial diagnosis. Four patients had a long-term recurrence. The median followup of the patients who had a recurrence was 15 years, and the median time to recurrence was 11 years. In all patients, the recurrence occurred in the neck; all but 1 patient had a recurrence after 9 years of follow-up.

At the time of the initial neck recurrence, 3 patients were treated with repeat surgery and 2 were on active surveillance, and none received additional RAI therapy. Of the 3 patients who were treated, 2 achieved no evidence of disease and 1 patient had a second neck recurrence by the end of the follow-up. The 2 patients on active surveillance after the initial neck recurrence continued to have stable persistent disease by the end of the follow-up. Thus, at the end of the follow-up period (median: 3 years), 1 had recurrent disease, 2 had persistent but stable structural disease, and 2 had no evidence of disease after repeat surgery.
La Greca/Xu/Ghossein/Tuttle/Sabra 
Table 4. Characteristics of the 5 patients who had a recurrence by end of follow-up

\begin{tabular}{|c|c|c|c|c|c|c|c|c|c|c|c|}
\hline $\begin{array}{l}\text { Age, } \\
\text { years }\end{array}$ & Sex & $\begin{array}{l}\text { Histology of } \\
\text { the primary } \\
\text { tumor }\end{array}$ & $\begin{array}{l}\text { Size of the } \\
\text { primary } \\
\text { tumor, } \mathrm{cm}\end{array}$ & $\begin{array}{l}\text { Vascular } \\
\text { invasion }\end{array}$ & $\begin{array}{l}\text { Extrathyroidal } \\
\text { extension }\end{array}$ & $\begin{array}{l}\text { Other } \\
\text { foci, } n\end{array}$ & $\begin{array}{l}\text { Size of the } \\
\text { other foci, } \\
\mathrm{cm}\end{array}$ & $\begin{array}{l}\text { Affected } \\
\text { N1b nodes }\end{array}$ & $\begin{array}{l}\text { Affected } \\
\text { Nla nodes }\end{array}$ & $\begin{array}{l}\text { Largest } \\
\text { affected } \\
\text { node }\end{array}$ & $\begin{array}{l}\text { Extranodal } \\
\text { extension }\end{array}$ \\
\hline 28 & $\mathrm{~F}$ & Classic & 2.5 & None & Absent & 2 & $<0.1-1.1$ & 11 & 0 & 2.5 & Yes \\
\hline 36 & $\mathrm{M}$ & Classic & 2.5 & None & Present & 4 & $<0.1-1.8$ & 41 & 11 & 1.8 & Yes \\
\hline 57 & $\mathrm{~F}$ & Tall cell & 4.5 & None & Absent & 2 & $0.4-2.5$ & 2 & 6 & 2.0 & Yes \\
\hline
\end{tabular}

$\mathrm{M}=$ Male; $\mathrm{F}=$ female; $\mathrm{N} / \mathrm{A}=$ not available.

Table 5. Other characteristics of the 5 patients who had a recurrence by the end of follow-up

\begin{tabular}{|c|c|c|c|c|c|c|c|}
\hline 290 & I & High & Biochemical incomplete & No evidence of disease & 23 & 11 & Neck \\
\hline 373 & I & Intermediate & Indeterminate & Structural persistent disease & 29 & 26 & Neck \\
\hline 152 & III & Intermediate & Excellent & No evidence of disease & 6 & 5 & Neck \\
\hline
\end{tabular}

\section{Discussion}

To our knowledge, this is the first study to describe the natural history of multifocal macroscopic papillary thyroid cancer without gross extrathyroidal extension. We found that the risk of recurrence in this cohort was low (6\%), similar to that in patients with multifocal microscopic PTC or that of classic papillary thyroid cancer (1$4 \mathrm{~cm}$ ) without extrathyroidal extension or nodal involvement. All of the recurrences were in the neck and none of our patients developed distant metastasis or died from thyroid cancer, supporting a low-risk behavior of this presentation.

As predicted, the vast majority of patients with multifocal macroscopic PTC (91\%) had no evidence of disease or an indeterminate response at the final follow-up. Almost all of the the patients with N0 disease had no clear structural evidence of thyroid cancer recurrence or persistent disease after the initial therapy or on follow-up, and none developed distant metastasis (fig. 1). When we examined patients with $\mathrm{N} 1$ disease, those at risk for distant metastasis were detected at diagnosis and none developed distant metastasis on follow-up. Also, 4 of the 5 patients who recurred had bulky nodal involvement in the lateral neck with extranodal extension at diagnosis. Therefore, as clinicians, we can easily identify early on in the disease process the small proportion of patients with multifocal macroscopic papillary thyroid cancer at risk for distant metastasis or a future recurrence.

As shown in tables 4 and 5, we observed that recurrence tends to occur late (the median time to recurrence was 11 years, with all but 1 patient having a recurrence after 9 years of follow-up), suggesting the need for longterm follow-up especially in patients presenting with bulky nodal involvement at diagnosis. One patient with multifocal macroscopic PTC without nodal involvement had a structural recurrence 2 years after his initial therapy after achieving a biochemical incomplete response. It can be argued that this patient had persistent disease after the initial therapy that went undetected and did not truly recur.

Our data are in concordance with most series reporting long-term follow-up of thyroid microcarcinoma patients $[17,18]$. In the paper by Baudin et al. [7] about thyroid microcarcinoma, multivariate analysis showed that the only 2 parameters that influenced recurrence were the 
number of thyroid microcarcinoma foci at pathologic examination and the extent of the initial thyroid surgery. Hay et al. [17] identified initial lymph node metastases and the extent of the initial thyroid surgery as the 2 risk factors for locoregional recurrence in a multivariate analysis of 535 patients with thyroid microcarcinoma [17]. In a prior series of 148 patients, examining the prognostic factors of papillary microcarcinoma, our group demonstrated that tumor multifocality, among other factors, correlated with the presence of metastatic nodes at diagnosis. In turn, the presence of significant nodal involvement ( $>3$ affected nodes) strongly correlated with a worse recurrence-free survival $(\mathrm{p}<0.001)$. One patient with poorly differentiated nodal metastasis died in that series [19]. In the current study of multifocal macroscopic PTC, we observed that almost all recurrences were also due to (or strongly interrelated with) bulky nodal involvement at diagnosis but did not correlate with the number of thyroid cancer foci.

Based on the review of the literature, the 2015 ATA risk classifier for structural disease specific recurrence (fig. 4 of the 2015 ATA guidelines) estimates that the risk of recurrences of unifocal intrathyroidal papillary thyroid microcarcinoma (1-2\%), multifocal papillary thyroid microcarcinoma (4-6\%), and intrathyroidal papillary thyroid cancer sized between 2 and $4 \mathrm{~cm}$ and irrespectively of multifocality $(\sim 5 \%)$ is low [10]. Our study suggests that patients with macroscopic multifocal PTC without gross extrathyroidal extension or clinically significant lymph node metastases can be similarly classified as 'low risk'.

We recognize that our study is limited by its retrospective design and the short median follow-up. Thus, we may have detected more recurrences if our cohort had been followed for more than 10 years. We propose that patients with multifocal macroscopic papillary thyroid cancer who present with bulky nodal involvement be treated with complete resection and RAI adjuvant therapy and remain under close follow-up for a long period of time (exceeding 15 years) as recurrences tend to occur late.

In conclusion, patients presenting with multifocal macroscopic papillary thyroid cancer without bulky nodal involvement or gross extrathyroidal extension have a low risk of thyroid cancer-specific recurrence and mortality.

\section{Acknowledgment}

This research was funded in part by NIH/NCI Cancer Center Support Grant P30 CA008748.

\section{Disclosure Statement}

No competing financial interests exist for Dr. Sabra. Dr. Tuttle is a consultant for Genzyme.

\section{References}

1 Russel WO, Ibanez ML, Clark RL, Withe EC: Thyroid carcinoma: classification, intraglandular dissemination, and clinicopathological study based upon whole organ sections of 80 glands. Cancer 1963;16:1425-1460.

2 Carcangiu ML, Zampi G, Pupi A, Castagnoli A, Rosai J: Papillary carcinoma of the thyroid: a clinicopathologic study of 241 cases treated at the University of Florence, Italy. Cancer 1985;55:805-828.

3 Clark RL, White EC, Russel WO: Total thyroidectomy for cancer of the thyroid: significance of intraglandular dissemination. Ann Surg 1959;6:859-866.

4 Mazzaferri EL: Management of low-risk differentiated thyroid cancer. Endocr Pract 2007; 13:498-512.

5 Roti E, degli Uberti EC, Bondanelli M, Braverman LE: Thyroid papillary microcarcinoma: a descriptive and meta-analysis study. Eur J Endocrinol 2008;159:659-673.
6 Chow SM, Law SC, Chan JK, Au SK, Yau S, Lau WH: Papillary microcarcinoma of the thyroid - prognostic significance of lymph node metastasis and multifocality Cancer 2003;98:31-40.

7 Baudin E, Travagli JP, Ropers J, Mancusi F, Bruno-Bossio G, Caillou B, Cailleux AF, Lumbroso JD, Parmentier C, Schlumberger M: Microcarcinoma of the thyroid gland: the Gustave-Roussy Institute experience. Cancer 1998;83:553-559.

8 Hay ID, Grant CS, Taylor WF, McConahey WM: Ipsilateral lobectomy versus bilateral lobar resection in papillary thyroid carcinoma: a retrospective analysis of surgical outcome using a novel prognostic scoring system. Surgery 1987;102:1088-1095.

9 DeGroot LJ, Kaplan EL, McCormick M, Straus FH: Natural history, treatment, and course of papillary thyroid carcinoma. J Clin Endocrinol Metab 1990;71:414-424.
10 Haugen BR, Alexander EK, Bible KC, Doherty GM, Mandel SJ, Nikiforov YE, Pacini F, Randolph GW, Sawka AM, Schlumberger M, Schuff KG, Sherman SI, Sosa JA, Steward DL, Tuttle RM, Wartofsky L: American Thyroid Association management guidelines for adult patients with thyroid nodules and differentiated thyroid cancer: the American Thyroid Association Guidelines Task Force on Thyroid Nodules and Differentiated Thyroid Cancer. Thyroid 2015;26:1-133.

11 DeLellis RA, Lloyd RV, Heitz PU, Eng C (eds): World Health Organization Classification of Tumours: Pathology and Genetics Tumours of the Endocrine Organs. Lyon, IAEC Press, 2004.

12 Ganly I, Ibrahimpasic T, Rivera M, Nixon I, Palmer F, Patel SG, Tuttle RM, Shah JP, Ghossein R: Prognostic indicators of papillary thyroid carcinoma with tall cell features. Thyroid 2014;24:662-670. 
13 Edge SB, Byrd DR, Compton CC, Fritz AG, Greene FL, Trotti A (eds): AJCC Cancer Staging Manual, ed 7. New York, Springer, 2009.

14 Tuttle RM, Tala H, Shah J, Leboeuf R, Ghossein R, Gonen M, Omry G, Fagin J, Shaha A: Estimating risk of recurrence in differentiated thyroid cancer after total thyroidectomy and radioactive iodine remnant ablation: using response to therapy variables to modify the initial risk estimated predicted by the new American Thyroid Association staging system. Thyroid 2010;20:1341-1349.
15 Momesso D, Tuttle RM: Update on differentiated thyroid cancer staging. Endocrinol Metab Clin North Am 2014;401-421.

16 Robbins RJ, Srivastava S, Shaha A, Ghossein R, Larson SM, Fleisher M, Tuttle RM: Factors influencing the basal recombinant human thyrotropin-stimulated serum thyroglobulin in patients with metastatic thyroid carcinoma. J Clin Endocrinol Metab 2004;89:60106016.

17 Hay ID, Grant CS, Van Heerden JA, Goellner JR, Ebersold JR, Bergstralh EJ: Papillary thyroid microcarcinoma: a study of 535 cases observed in a 50-year period. Surgery 1992;112: $1139-1147$
18 Mazzaferri EL, Jhiang SM: Long-term impact of initial surgical and medical therapy on papillary and follicular thyroid cancer. Am J Med 1994;97:418-428.

19 Ghossein R, Ganly I, Biagini A, Robenshtock E, Rivera M, Tuttle RM: Prognostic factors of papillary microcarcinoma with emphasis on histologic subtyping: a clinicopathologic study of 148 cases. Thyroid 2014;24:245253 